\title{
EEG alpha entrainment reaction within the biofeedback setting and some possible effects on epilepsy
}

\author{
LARRY ROUSE, JOHN PETERSON, and GARY SHAPIRO \\ Biophysics Laboratory, California State University, Fresno, California 93740
}

\begin{abstract}
Twenty-four human volunteers were given one 30-min EEG alpha biofeedback training session within an active placebo setting. Ten dependent measures computed from serial 3-min period distributions showed no difference between contingent and yoke conditions or between 9-11 and 8-13 $\mathrm{Hz}$ feedback. Most subjects showed entrainment of the alpha rhythm toward a stable frequency over trials. Five additional subjects given five daily 30-min sessions of 9.5-10.5 Hz feedback showed a repetition of this same effect. Over a period of 4 months, one grand mal epileptic gradually entrained a bimodal period distribution toward a unimodal one centered at $10 \mathrm{~Hz}$. This effect was paralleled by a significant drop in seizure rate. These results were interpreted in terms of electrophysiological homeostasis.
\end{abstract}

Several experiments have reported that human subjects have used information provided by an EEG alpha/no-alpha biofeedback loop (Mulholland \& Runnals, 1961) to manipulate the occurrence of alpha waves $(8-13 \mathrm{~Hz})$ vs little or no alpha (Beatty, 1971, 1972; Brown, 1970, 1971; Hord \& Barber, 1971; Hord, Naitoh, \& Johnson, 1972; Nowlis \& Kamiya, 1970; Peper, 1970, 1971, 1972, Peper \& Mulholland, 1970). No one to date, however, has shown that subjects can learn to increase alpha abundance above that found diring maximal baseline conditions (Lynch \& Paskewitz, 1971; Paskewitz \& Orne, 1973), show "nonmediated" control or produce more alpha than noninstrumented techniques such as hypnosis, meditation, or autogenic relaxation. Most have reported that subjects can much more easily reduce alpha than increase it.

Most studies have exclusively relied upon within-subject measurements as an index of "alpha control." Only one experiment (Engstrom, London, \& Hart, 1970) has reported a between-group comparison using taped (standard pattern) yoked controls, but unfortunately did not match subjects on the basis of initial baseline alpha index (percent time) and used an analysis of covariance to adjust measurements taken after alpha feedback training. Although such factors as skull thickness affect alpha amplitude (Leissner, Lindholm, \& Petersen, 1970), subjects with initially low baseline alpha index show larger relative increases over time independent of response differentiation (Beatty, 1971, 1972; Lynch \& Paskewitz, 1971). The use of equal random or matched subject groups and comparisons between contingent and yoked controls seems essential to the study of the operant conditioning of EEG alpha activity.

Mulholland (1972) has operationally defined alpha as an $8-13-\mathrm{Hz}$ signal of at least $25 \%$ of its maximum baseline amplitude recorded from the posterior regions of the scalp. All of these parameters have varied significantly in the above-mentioned experiments and determine the estimation of the percent-time alpha index (Boudrot, 1972). Also, set and setting are undoubtedly important factors operating on the EEG independently of the feedback loop itself (Timmons, Salamy, Kamiya, Girton, 1972). In fact, in one recent investigation (Beatty, 1972), placebo reactions due to instructions given to each subject before feedback were found to account totally for the magnitude and direction of change in alpha index with or without feedback. The fact that the frequency-transfer function of the feedback loop interacted with an opposing placebo reaction in subjects who received false instructions, however, supports the existerice of a parallel effect of feedback and programmed mediation and suggests the use of yoked controls in the study of feedback control.

Several papers have also offered data which suggest that subjects might actually manipulate different frequency bands within the clinical EEG range and within the alpha band in particular, thereby significantly altering physiological functions (Brown, 1970, 1971; Green, Green, \& Walters, 1970; Kamiya, 1969; Sittenfeld, 1972; Sterman, 1973; Sterman \& Friar, 1972). Likewise, several have reported that meditation produces an increase in a persistent, topographically synchronous, and stable alpha frequency at or near $10 \mathrm{~Hz}$, depending upon the style and level of meditation and particular subject (Banquet, 1973; Timmons \& Kamiya, 1970; Wallace \& Benson, 1972).

In contrast to the theory that alpha represents an "off," inhibited or idling brain state, Brumlik, Richeson, \& Arbit (1966) reviewed experiments that demonstrated alpha to be significantly related to various metabolic and nervous functions involved with "on," excited or active states. Andersen and Andersson (1968) also argued that 
alpha was an active process involved in sensory recognition, storage, and retrieval functions.

Many psychophysiological measurements have been successfuly mapped onto various parameters of the alpha rhythm. This, plus the substantiated viewpoints of alpha as a dynamic nervous process (Nebylitsyn, 1972) involved in homeostasis (cf. Gullickson, 1973), support the existence of an equilibrium point represented by a frequency and amplitude window at or very near exact $10 \mathrm{~Hz}$.

Increased alpha synchronization may be viewed as an entrainment phenomenon during which local oscillations in the brain pull together toward a dominant frequency (Dewan, 1969). Mulholland's (1972) view of the response of alpha to external sensory feedback as a time series of a disturbance function that returns to a steady state supports the prediction that alpha frequency stabilization at or toward $10 \mathrm{~Hz}$ will occur in response to alpha feedback training. Mimura, Sato, Ozaki, Honda, \& Masuya (1962), in fact, found this to occur as tones were repeatedly used to block the alpha.

The present study attempted to compare the entrainment phenomenon occurring during alpha feedback to that independent of the loop. Narrow vs wideband alpha feedback was also studied as a first effort toward relating the frequency-feedback range in the loop to the range of alpha frequencies produced. The main effect involved measures of frequency stabilization and points toward an objective index of "centering."

\section{METHOD}

Subjects were 24 undergraduate students (ages 17-35) given credit for participating in the experiment. Twelve male and 12 female subjects were randomly and equally assigned to a 2 by 2 factorial design with repeated measures. One group received 9-11 Hz feedback, a second received the usual 8-13 Hz feedback. Two remaining groups received taped-yoke stimulation on a paired contingent to yoke subject basis. Five additional subjects volunteered for longer-term training. Four trained on 9.5-10.5 Hz feedback for 5 days, $30 \mathrm{~min}$ per day. One grand mal epileptic trained four sessions per week for 1 week on $8-13 \mathrm{~Hz}$ and then for 4 months on $9-11 \mathrm{~Hz}$. Seizure rate was $1 /$ week at the beginning of training. The patient was under the influence of daily anticonvulsant medication throughout the experiment.

The EEG was amplified by a Grass Model III-A electroencephalograph interfaced with a Krohn-Hite variable bandpass filter (Model 3550-R) set for half-amplitude at 6 and $16 \mathrm{~Hz}(24 \mathrm{~dB} /$ octave attenuation slope) and maximally flat between $8-13 \mathrm{~Hz}$. On-line period analysis was performed by an electronic system to be described in detail elsewhere (Rouse, in press). The major advantage of this system is its preservation of signal time above threshold (e.g., alpha index) in terms of counts or number of half waves per epoch while remaining insensitive to signal-amplitude variation with respect to frequency computed on the basis of time between baseline crossings (Creutzfeldt, Greenwald, Simonova, \& Schmitz, 1969). Amplitude threshold for this experiment was just above the noise level and therefore yielded data inclusive of, but not equivalent to, the percent-time alpha index based upon higher voltage criterion. A single-channel frequency analyzer with step-function cutoffs $(.1 \mathrm{~Hz}$ resolution) controlled the high- and low- $(\mathrm{RC}=.25 \mathrm{sec})$ amplitude alpha feedback tones. A multichannel analyzer (Nuclear Data) continuously accumulated both positive and negative half-wave periods over each 3 -min epoch and visually displayed the subsequent period distributions with $.5 \mathrm{~Hz} /$ channel resolution. Memory-typeout data was transferred to computer punch cards for parameter calculations to be used in subsequent statistical analyses. Four spectra were taken from each subject: (1) prefeedback baseline, (2) after $10 \mathrm{~min}$ of feedback (or yoke stimulation), (3) after $20 \mathrm{~min}$ stimulation, and (4) immediately after feedback was terminated. A set of 10 repeated measures was compiled by a CDC 3150 digital computer from the counts $/ \mathrm{Hz}$ typeout $(7-14 \mathrm{~Hz})$ from each spectrum. This range contained from 3,000 to 4,000 half-wave measurements. The repeated measures were: mean and modal frequency, variance, modal counts, $10-\mathrm{Hz}$ counts $( \pm .25 \mathrm{~Hz}), 9-11-\mathrm{Hz}$ counts, $8-12-\mathrm{Hz}$ counts, $7-14-\mathrm{Hz}$ counts, kurtosis index ( $\mathrm{K}=$ modal counts/variance), and centering index $(\mathrm{C}=10-\mathrm{Hz}$ counts/variance). The last two measures described the general shape of each distribution. Individual data were graphed and inspected for each subject. A two-way ANOVA with repeated measures was then performed on each of the 10 measures. Separate one-way ANOVAs with repeated measures checked for sex differences. All Fortran IV programs are available upon request.

Grass silver-silver chloride cup electrodes were at tached by Grass electrode cream and tissue paper to a point $2 \mathrm{~cm}$ to the left of $C_{z}$ and referenced to the contralateral earlobe $\left(A_{2}\right)$ with the ipsilateral earlobe grounded. Subjects reclined with eyes closed in total darkness on a soft cot in an electrically shielded room (6 3 meters). Subjects were free to open eyes infrequently, for comfort. Two feedback tones (both $160 \mathrm{~Hz}$ ) were controlled by the contingent subject's alpha rhythm. One tone on designated alpha within the frequency limit and above 10 microV. Two tones on meant alpha above 20 microV. The epileptic trained in the presence of a continuous background $80-\mathrm{Hz}$ tone and dim light with eyes open/close option to facilitate concentration and relaxed wakefulness. At the end of each session, the patient was shown the four period distributions taken during training and then given an appraisal of the degree of centering that day and relative to past performance. This patient had difficulty with drowsiness during the first month of training in total darkness and was subsequently shifted to a brighter condition. A $320-\mathrm{Hz}$ tuning fork was used by this patient to practice mantra meditation (Banquet, 1973) outside the lab.

A strong effort was made tovard establishing a uniform and positive set and setting that would bring out any placebo reaction in the yoked controls and to reduce data variablity due to this factor. Subjects were given an instruction and information form to complete 1 week before the experiment, scheduled to occur in the evening hours. The instructions informed each subject that volitional control of alpha brainwaves was possible and that the following suggestions borrowed from meditation and feedback literature would give them a head start: (1) Don't try too hard; maintain a neutral attitude toward the task, i.e., don't prejudge what will happen. (2) Maintain a balanced level of consciousness midway between drowsiness and arousal, i.e., relax but don't go to sleep. (3) Don't fix on any particular imagery; let the mind flow freely. (4) Concentrate on the tones as internal experience, i.e., sound in your head; let the outside tones reinforce this. Subjects were questioned about drowsiness after each training session.

\section{RESULTS}

There were no sex differences on any measure, nor were there between-group differences that could be attributed to factors other than chance or those present during prebaseline. All groups were statistically equal at prebaseline on all measures except mean and modal frequency and $7-14-\mathrm{Hz}$ total counts. Several measures did change over time, however, indicating the presence 
of a strong placebo reaction. Total group average mean frequency gradually decreased by $.2 \mathrm{~Hz}(\mathrm{p}<.01)$, group average modal frequency gradually decreased by $.1 \mathrm{~Hz}$ $(p<.05)$. These two measures ranged for all subjects between 9.5 and $10.5 \mathrm{~Hz}$. Group average mean frequency asymptote at postbaseline was $10.05 \mathrm{~Hz}$. Frequency slowing was the strongest effect found, observable clearly in all but four subjects. Seven out of eight subjects reporting drowsiness during the session showed an exaggerated slowing and shift toward the bimodal alpha-theta distribution typical of sleep onset and deprivation (Williams, Granda, Jones, Lubin, \& Armington, 1962). These subjects also showed the largest decreases in $\mathrm{K}$ and $\mathrm{C}$ indexes. Half of the remaining subjects not reporting drowsiness showed extremely large increases in $\mathrm{K}$ and $\mathrm{C}$, some subjects doubling them. Figure 1 illustrates the slowing effect and four types of entraining responses all of which are associated with rapdily increasing $\mathrm{K}$ and $\mathrm{C}$.

Figure 2 shows the incidence of activity in four successively inclusive bandpasses averaged over all subjects and plotted as a function of time. The increase in counts over time is significant for every band $(\mathrm{p}<.05)$ except for $8-12 \mathrm{~Hz}$. This pattern results from most subjects entraining toward a center frequency with a consequent decrease in adjacent channels. The more peripheral channels increase as a consequence of activity outside the bandpass also being pulled toward center. The $\mathrm{K}$ and $\mathrm{C}$ indexes mentioned above both show a trend toward increasing $(p<.1)$ when analyzed across trials within all 24 subjects. If the eight subjects reporting drowsiness are dropped, this analysis yields a significant change $(p<.05)$. Group analysis of period distribution variance showed no significant change over time although this is clearly not the case for several individuals, including the drowsers. The fact that activity outside the $7-14-\mathrm{Hz}$ band is also pulled toward the peak frequency accounts for the lack of change in variance computed on the basis of activity inclusive of this band.

Results from the 1-week trainers showed no significant gain over that achieved by the best subjects in the first experiment. They did, however, show an interesting alternation on successive days between entrainment on vs off $10 \mathrm{~Hz}$. One subject with high $\mathrm{K}$ showed at Day 1 baseline a bimodal distribution peaking at 9 and $11 \mathrm{~Hz}$. The two major components could easily be seen in the filtered EEG which showed a high instability of alpha frequency within individual alpha spindles. Step changes on the order of $2 \mathrm{~Hz}$ and greater were frequent. During training, this particular subject was able to entrain this pattern into a unimodal distribution centered at $10 \mathrm{~Hz}$ with increased $\mathrm{C}$. The number of $10-\mathrm{Hz}$ counts was gradually increased by $100 \%$ over the training trials.

The epileptics baseline distribution was strongly bimodal with peaks at 6.5 and $13 \mathrm{~Hz}$ and with a very weak and inconsistent peak at or near $10 \mathrm{~Hz}$. Average
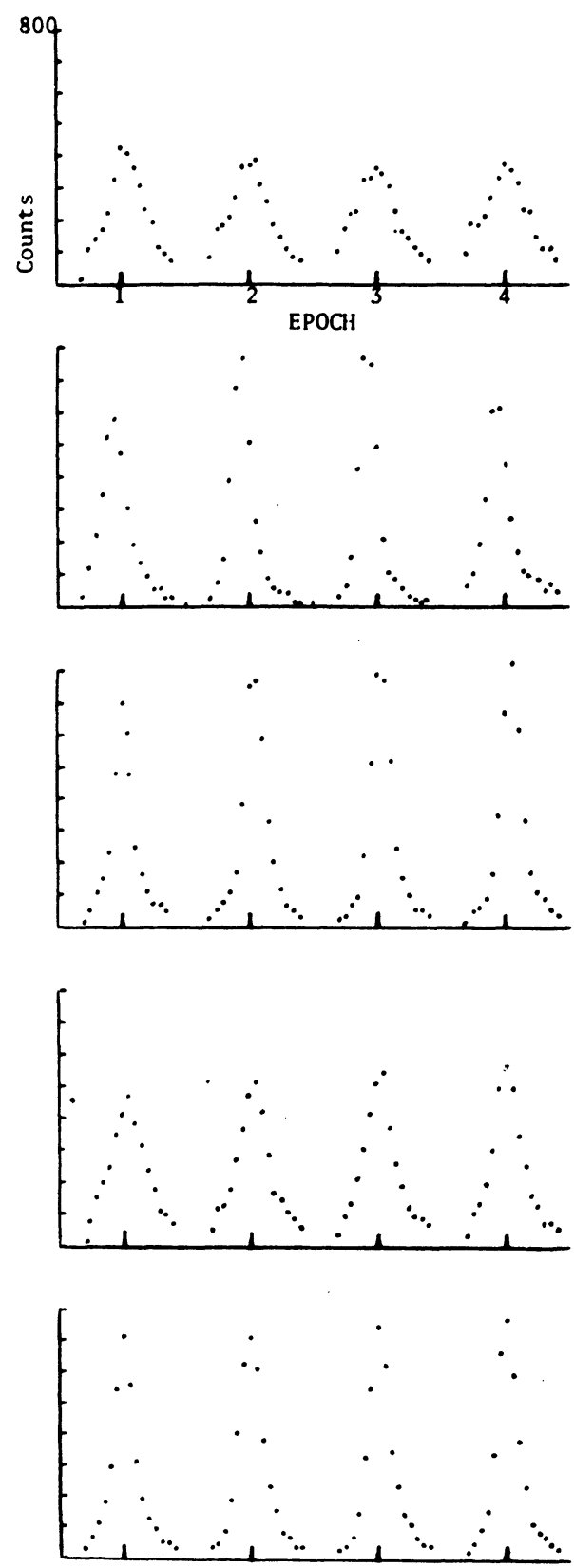

Figure 1. Each graph contains four successive 3-min period distributions, one before, two during, and one after yoke or feedback stimulation. The midmarkers are at $10 \mathrm{~Hz}$ for each distribution with $.5 \mathrm{~Hz} /$ channel resolution. The top series is typical of the frequency slowing and decreased alpha entrainment associated with drowsiness and onset of sleep. The bottom four show different modes of entrainment in the alpha band; one below, one above, one onto, and one at $10 \mathrm{~Hz}$.

amplitude was above 10 microV for all three points in the distribution as indicated by the segregated period distribution (Rouse, in press) of activity above this threshold (spectra from the nonepileptics showed an opposite trend toward peaking at one frequency as amplitude threshold was increased). This pattern 


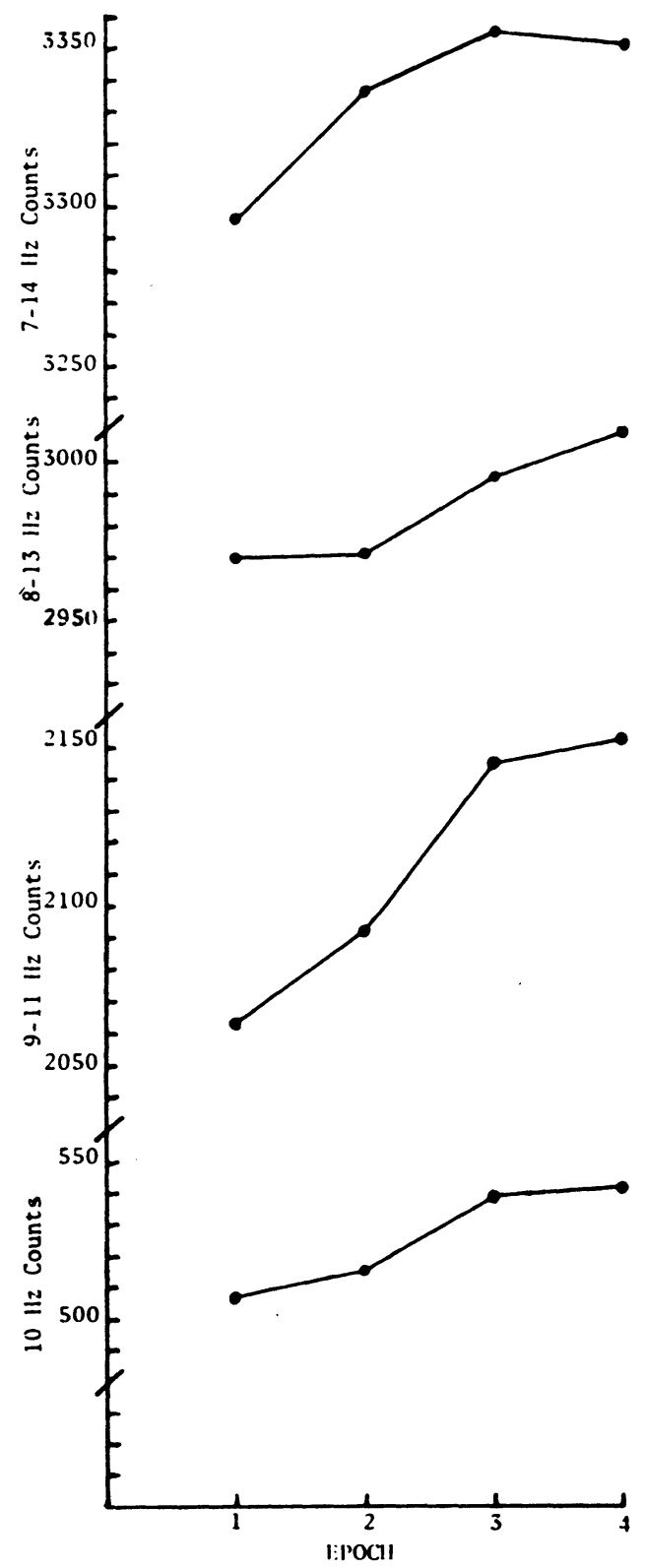

Figure 2. Total group averaged counts within four successively inclusive frequency bands plotted as a function of time. All bands significantly increased except for 8-12 Hz, indicating entrainment toward a central frequency.

remained unaltered for 2 months except for very slight and unstable trends toward entrainment at and near $10 \mathrm{~Hz}$ during feedback, which persisted during postfeedback baseline but disappeared overnight. During the third month, this change began to appear in the prefeedback baselines and continued to increase during training, decreasing slightly at postbaseline. At this point, the $8-13-\mathrm{Hz}$ bandpassed period distribution was peaked at $10 \mathrm{~Hz}$. Entrainment was always accompanied by count reduction in the bimodal peaks. Figure 3 shows this progression of events which was associated with improvement in the clinical condition of the patient who was seizure free during the last $1-1 / 2$ months of training and has remained so for 18 months after termination of training but with continued daily tuning-fork practice. This patient's history has included seizure-free periods as long as 6 months with anticonvulsant medication. Seizures occurred in prolonged bursts at $1 /$ week. There was, therefore, a strong probability for a positive placebo reaction. The EEG correlate of this reaction parallels that found with nonepileptic subjects.

\section{DISCUSSION}

The apparent lack of effect due to short-term feedback independent of a strong effect due to instructions to the subject should reemphasize the importance of including yoked controls and careful design in instructions. Differences at baseline and changes over time in parameters of the period distribution point out the need for carefully matched groups and caution against overgeneralizing results on the basis of single-band analysis even within the traditional alpha-frequency range. However, these data do not exclude a covert effect of the feedback loop on alpha frequency stabilization. Our current approach to proving has added augmented frequency feedback to inform the subjects of where and how far off center they are when the tones are off.

The general slowing of alpha frequency gradually over time in the relaxing subject is a well-known phenomenon first reported by Hans Berger in 1939. Slowing in the feedback loop has been reported to range from $.1-1 \mathrm{~Hz}$ (Brown, 1970; Hord et al, 1972) and is associated within limits with reduced CNS arousal (Creutzfeldt et al, 1969; Lynch \& Paskewitz, 1971) and increased percent time alpha (Anderson \& Andersson, 1968; Nebylitsyn, 1972) both of which frequently occur within the biofeedback and meditation settings.

The occurrence of alpha is classically associated with relaxed wakefulness, an equilibrium point between arousal and sleep (Darrow, 1947; Gloor, 1969; Lynch \& Paskewitz, 1971). There also exists measurable arousal and reaction time continua within alpha. These are related through an inverted U-shaped curve to alpha amplitude (Stennett, 1957) and a continuously increasing function to frequency (Nebylitsyn, 1972; Surwillo, 1963). Single alpha spindles are also probabilistically associated with successful recall (Darrow, Vieth, \& Wilson, 1957). Past confusion of orienting behavior with later components of attention has generated some thought about alpha and attention being incompatible. It now seems that a dispersion of multiple alpha generators from a modal frequency occurs during orienting and that beta frequencies are actually attentuated during this process (Mimura, et al, 1962). Postorientation synchronization of the alpha rhythm appears to be involved in recognition, storage and retrieval processes (cf. Andersen \& Andersson, 1968; Brumlik et al, 1966, 1967). 

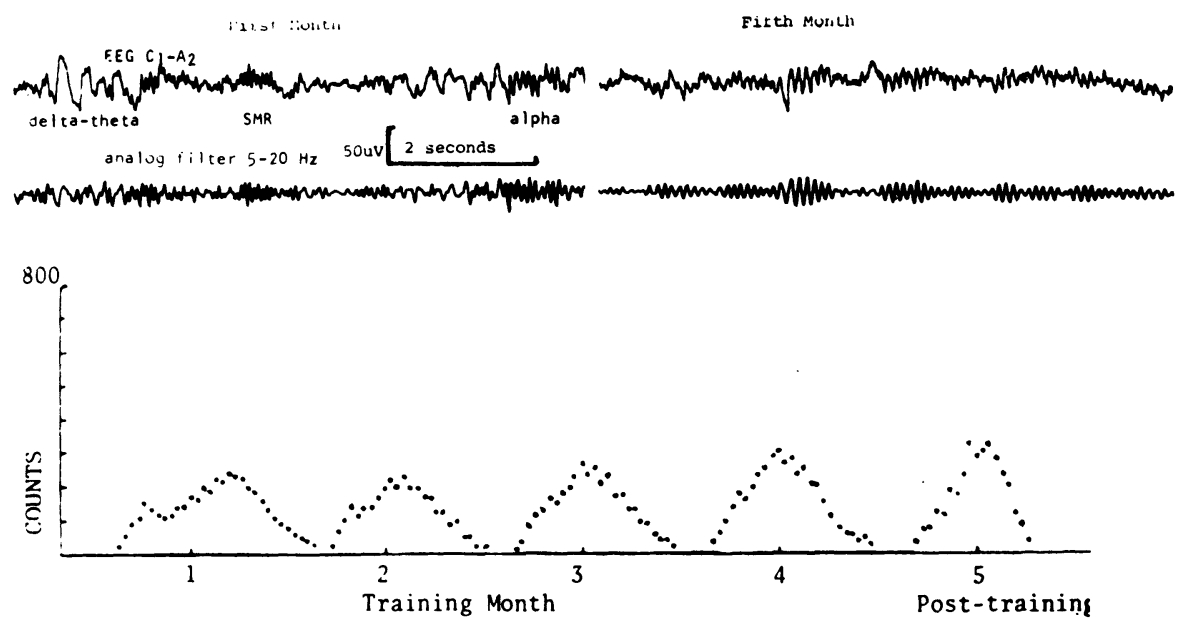

Figure 3. Four representative distributions produced by a grand mal epileptic who trained regularly for 4 months on a 9-11-Hz feedback loop. Gradual entrainment toward $10 \mathrm{~Hz}$ was associated with significant improvement in the patient's clinical condition and parallels the active placebo entrainment response of nonepileptic subjects to shortterm exposure to the biofeedback setting.
A general inverse relationship between frequency and both amplitude and alpha duration was reported as a basic physiological property of the alpha rhythm (Andersen \& Andersson, 1968). Increased habituation during longer alpha trains as described by the alpha attentuation cycle (Mulholland, 1972) also occurred during slower alpha frequencies below $10 \mathrm{~Hz}$ as compared to faster ones above $10 \mathrm{~Hz}$ (Nebylitsyn, 1972). Andersen, Junge, \& Sveen (1972) recently showed that cortical synchronization (by direct electrical stimulation) facilitates thalamic transmission. Latency reduction was optimal at frequencies between 10 and $20 \mathrm{cps}$. Alpha synchronization during habituation or reduced cortical input in general, then, appears to be directly involved in increasing the gain in thalamocortical transmission. Synchronization below $10 \mathrm{~Hz}$ is suboptimal in compensating for reduced transmission and therefore might be directly involved in the habituation process itself. These data are consistent with Lindsley's (1961) finding that conduction time for unimpaired recognition in the human primary visual pathway corresponds to $12.14 \mathrm{~Hz}$, Slatter's (1960) finding that visual imagery is correlated with $12 \mathrm{~Hz}$ (while all other alpha frequencies are decreased or uncorrelated), Howe and Sterman's (1973) finding that the $12.14 \mathrm{~Hz}$ sensorimotor rhythm (SMR) occurs during a gated disinhibition of the somatosensory pathway, and Morrell's report (1966) that stimulus-provoked alpha is higher in frequency, amplitude, and regularity than background alpha.

On a basic level, $10 \mathrm{~Hz}$ seems to be an optimal point of electrical operation for certain nervous functions. Transfer functions through thalamic neuron cell membranes (Andersen \& Andersson, 1968), nuclei (Killam \& Killam, 1957) and the primary visual pathway (Perry \& Childen, 1969) center at or very near $10 \mathrm{~Hz}$. It is potentially the most efficient "clock frequency" (Klir, 1972; Rubin \& Wells, 1969) and the continuum within which it operates represents a fundamental dynamism (excitation-inhibition) of the nervous system (Nebylitsyn, 1972). Alpha frequency has also been negatively correlated with response automization (Vogel,
Broverman, \& Klaiber, 1968), resistance to photic driving (Vogel \& Kun, 1969a), increased androgen (Vogel, Broverman, Klaiber, Abraham, \& Cone, 1971a) and estrogen (Vogel, Broverman, \& Klaiber, 1971b) and positively correlated with IQ (Giannitrapani, 1969, 1973; Vogel et al, 1968, Vogel, Kun, Meshour, Broverman, \& Klaiber, 1969b).

There is, therefore, ample reason to believe that alpha frequency stabilization at or near $10 \mathrm{~Hz}$ does represent an actual balance or centering point and further that this homeostatic mechanism is a general one shared by more than one thalamocortical system. Because of the diversity of these relationships, it may prove useful to call entrainment toward or at $10 \mathrm{~Hz}$ centering and index its degree by the ratio: $(10 \mathrm{~Hz} \text { counts })^{2} /($ variance $x$ modal counts). Increased $C$ values due to entrainment near but not toward $10 \mathrm{~Hz}$ would be reduced by the ratio $\mathrm{C} / \mathrm{K}$. Since there seems to be an optimal amount of alpha for attention (Creutzfeldt et al, 1969; Legewie et al, 1969), there is probably an optimal degree of entrainment. Analytical study of an entrainment continuum is therefore needed. [A rebound entrainment is reportedly typical after a "physically challenging experience" (Adey, 1969).] This process is related to the well-known "post-reinforcement synchronization" phenomenon (Sterman, Wyricka, \& Roth, 1969) which indicates that the overall reinforcement value of the biofeedback setting is an important factor. Increased stimulus complexity increases alpha abundance if the stimulus is pleasant but decreases it if unpleasant (Gale, Bramby, Lucas, \& Christie, 1972). Sterman (1972b) found that subjects who reported enjoying alpha biofeedback produced more alpha; those who disliked it did not. All such factors then should interact to form the subject's response to the feedback setting as well as any contingency present.

The positive correlation between percent-time and maximum amplitude of alpha and the negative correlation between percent-time and frequency are contradicted by some reports of a zero order correlation between amplitude and frequency (Nebylitsyn, 1972). However, the entrainment index is also an increasing function of 
alpha amplitude threshold used to segregate different period distributions. These relationships remain uncontradicted if we assume the existence of an additional continuum within the alpha rhythm that could interact with the arousalsleep continuum to produce a higher net system activation during optimal amplitudes as demanded by the U-shaped curve. This continuum could be related to sustained attention (as distinguished from the orienting and arousal reactions) and described in terms of the Entrainment Index $\mathbf{K}$. This relationship is supported by earlier reports of alpha entrainment during some mental operations demanding sustained attention (Kreitman \& Shaw, 1965).

The several "active" models of alpha corroborate the entrainment-attention hypothesis: (1) alpha as a carrier wave for transmission between the cortex, thalamus, and brainstem systems (Andersen \& Andersson, 1968); (2) as a process identification test stimulus (Dewan, 1969); (3) as a gate process involved in relaxing sensory suppressor zones with consequent transmission of primary sensory data from konico to isocortex and consequently into conscious perception (Stanley-Jones, 1970); (4) as an inherent rhythm involved in holographic memory process (Pribram, 1969; Walter, 1969).

These models must be viewed as complimentary descriptions of functions operating at different levels of organization within the brain. Alpha recorded in the EEG is not functionally equivalent to postsynaptic potentials of the same period, for example. Since there are fundamental signal sources for alpha in the cortex at the synaptic level and there are phase, frequency, and amplitude differences between "cortical columns" not larger than $.5 \mathrm{~mm}$ in diam (Andersen \& Andersson, 1968), thousands of different alphas with different frequencies, amplitudes, and phase relations occur simultaneously beneath a single surface electrode and are averaged through the scalp as the EEG, a highly processed signal formed through the process of entrainment and averaging (Delucchi, Garoute, \& Aird, 1962; Dewan, 1969; Perez-Borja, 1962).

Andersen and Andersson (1968) proposed that these signal generators are entrained by individual cell groups within the thalamus which as a whole acts as a master pacemaker among all the facultative pacemakers. A central entraining mechanism would subserve an integrating function within and between the specific and nonspecific thalamic nuclei. A dual entrainment process therefore exists, one specific and topographically restricted and one nonspecific and having an influence topographically more widespread. Lehmann (1971) has reported the existence of three major "functional lobes" of the brain with respect to alpha coherence. However, as entrainment occurs, the intraspindle frequency of a larger and larger area of cortex stabilizes to that of the common pacemaker. Further, a constant phase relation between different parts of the cortex occurs during this process (Adey, 1969; Fehmi, 1971) and has been reported to occur during alpha biofeedback training (Hord et al, 1972) and meditation (Banquet, 1973). That sensory integration does occur during entrainment is supported by a report that increased interhemispheric in phase stabilization in the feedback loop was mediated by "attentional focusing on a relatively stable image" (Fehmi, 1971) and by the relationship of entrainment to alertness within a context of relaxation.

The orienting reaction classically involves the temporary disruption of entrainment in the specific and nonspecific systems in preparation for reintegration. Because entrainment involves intra- and interhemispheric phase stabilization, the "bipolar" recording technique would tend to show less "alpha" during entrainment than would be detected by the "monopolar" technique which uses a relatively inactive reference. This effect helps to clarify why many studies have contradicted one another on the alpha-attention problem (Creutzfeldt et al, 1969).

Along related lines, the relation of function to topographical location is another important problem to consider. For example, recent neurophysiological evidence indicates that the inferior temporal lobes control visual processing during the orienting reaction and that the occipital lobes are disentrained at this early point (Pribram, 1969). Chapman, Armington, \& Bragdon (1962) reported that temporal-lobe alpha activity (called kappa activity) increased during intellectual tasks while occipital alpha did not. The eyes open-closed effect seems to be a parallel but separate phenomenon.

As shown in Figure 1, subjects entrain at various frequencies at and around $10 \mathrm{~Hz}$-some slowing, some increasing in mean frequency, most decreasing. Some subjects produce bimodal and even multimodal distributions during an epoch as long as $3 \mathrm{~min}$. The epileptic distribution is at the extreme minimum of entrainment at $10 \mathrm{~Hz}$. The group average moved toward exact $10 \mathrm{~Hz}$ as did the majority of individuals (those who did not drowse). There is then a continuous line of evidence describing $10 \mathrm{~Hz}$ as a functional equilibrium point within what Morrell (1966) has called a "continuum of consciousness."

The use of entrainment measures has previously been suggested for the study of the functional integration of various subsystems within and between the hypothalamus, thalamus, and various reticular nuclei (Hernandez-Peon, 1963). With regard to the latter, the lateral norepinephrine mediated arousal system, medial serotonin mediated depression system and lateral Ach mediated attention system have been proposed to ascend in parallel from the brainstem reticular complex to synergistically control the continual sensory processing that occurs within the specific pathways (Jasper, 1969). The arousal and depression systems are reciprocally inhibitory (Mabry \& Campbell, 1973). The Ach ascending system connects with the nonspecific thalamus and diffusely projects through specific nuclei of the thalamus to topographically specific but widespread areas of the cortex. Since cortical 
desynchronization is not necessary for alertness, attention, or learning and because late components of the evoked potential reflecting these three phenomena are suppressed by atropine sulfate, the Ach system is most likely involved in the alpha entrainment process. In this way vigilance and attention were considered to be separate but interacting continua (Jasper, 1969). Additional experimental tests of this theory may confirm Andersen and Andersson's (1968) inferred hypothesis that alpha reflects an active process necessary for higher learning to occur and could lead to valuable improvements in learning and therapy techniques.

The original theory of the centrencephalic system (Penfield \& Jasper, 1954), proposed that the highest level of integration was to be found within the brainstem reticular system. The basal forebrain region has also been proposed to be a high-level integration center in two-way communication with widespread areas of the brain (Sterman \& Clemente. 1968). Because alpha is such a predominant and widespread rhythm, more precise and encompassing electrophysiological measures such as alpha entrainment will definitely facilitate further study of brain function along these lines.

Reports on the relation of alpha index to seizure susceptibility have been contradictory (Friedlander, 1962) but failed to include entrainment measures. The gradual development of "perfect" alpha spindles (stable frequency and sinusoidal modulation, i.e., those brought about by entrainment) has been reported to accompany the elimination of seizures by anticonvulsant medication (Delmas-Marsalet, 1964). Alpha biofeedback is reportedly being used to treat epilepsy with some success (Poirier, 1973). Although there is much more work to be done in proving this effect, there is a very sound physiological basis for believing it to exist in some form. Hans Berger (see Gloor, 1969) originally related integrity of the alpha rhythm to the maintenance of a balanced consciousness and elevated seizure thresholds in human subjects. It should also be noted that Darrow (1947) related homeostatic regulation to both EEG alpha production and resistance to epileptic seizures.

It was reported here that long-term training in the feedback setting by an epileptic initially deficient in $10-\mathrm{Hz}$ entrainment was paralleled by a gradual entrainment toward this point over a period of 4 months and was also accompanied by a large drop in seizure incidence. Similar therapeutic effectiveness in an epileptic has been reported for feedback training of a high alpha variant (12 Hz SMR) recorded from the sensorimotor region of the cortex (Sterman \& Friar, 1972; Finley, Smith, \& Etherton, in press.) This latter technique is thought to exercise inhibitory functions peculiar to the specific pathways within the sensorimotor system rather than involve mutural entrainment by the nonspecific system (Sterman, 1973). Theoretically, entrainment in a specific thalamocortical system engages nonspecific integration (Andersen \& Andersson, 1968) and leads to similar clinical changes. Likewise, nonspecific entrainment should exercise the specific system. Future therapy might apply the two kinds of feedback to different types of epilepsy or combine them with new techniques to form a more effective therapy.

Fink (1963) and Fink and Itil (1968) reported that approximately 1 microgram $/ \mathrm{kg}$ of d-LSD-25 given IV to a grand mal epileptic $24 \mathrm{~h}$ after a convulsion eliminated postconvulsive slowing in the EEG in $40 \mathrm{~min}$ and produced an increased synchronization at $10 \mathrm{~Hz}$ $\left(100\right.$ microV, $\left.F_{2}-0_{2}\right)$. The exact frequency of the dominant alpha rhythm produced in epileptics given $1 \mathrm{microgram} / \mathrm{kg}$ has varied (see references in Fink \& Itil, 1968). This entrainment reaction is found at dose levels well below that which would be expected to produce a direct anticonvulsant effect (Rouse \& Frank, 1974) and has been generally found to accompany normalization of the epileptic's unstable EEG (Fink \& Itil, 1968).

Although some reports (e.g., Walter, W. Grey, personal communication; Cooper, Shipton, Shipton, Walter, \& Walter, 1957; Rodin \& Luby, 1966) have found that moderate (approximately $1 \mathrm{microgram} / \mathrm{kg}$ ) to high (much greater than 1 microgram $/ \mathrm{kg}$ ) dosages attenuate the amplitude and accelerate the frequency of the human EEG alpha rhythm, related work found this effect to vary with subjects' set and setting (Bradley, Elkes, \& Elkes, 1953; Brown, 1968). Guiti (1966) found that the alpha enhancement reaction to 1 microgram $/ \mathrm{kg}$ in dogs was accompanied by behavioral relaxation. Itil (1969) found that the dominant EEG frequency had upshifted to exactly $10 \mathrm{~Hz} 46 \mathrm{~min}$ after $1 \mathrm{microgram} / \mathrm{kg}$ (IV). This reaction only occurred in subjects who responded positively to the drug. Compared to baseline conditions, this EEG change showed reduced frequency and amplitude variability, i.e., the alpha rhythm had centered, not permanently desynchronized or disappeared. Schizophrenics are reported to show a more variable and slower alpha rhythm compared to normals (Giannitrapini, 1974). Oral administration of d-LSD-25 at $1 \mathrm{microgram} / \mathrm{kg}$ or higher usually produces a late episode of desynchronization in naive subjects who become aroused or tense. This effect is related to the orienting response and is nonspecific to LSD [e.g., amphetamine (Fink \& Itil, 1968)] . At .3 microgram $/ \mathrm{kg}$ the alpha rhythm is stabilized without amplitude reduction (e.g., Pfieffer, Goldstein, Murphree, \& Sugerman, 1965). This latter dosage is at the low end of the minimally effective dose range (20-100 micrograms) for alteration of consciousness in naive human subjects (Goodman \& Gilman, 1968). Alpha enhancement accompanies a positively felt mood change (within the context of relaxed wakefulness) after drug administration in general (Fink, 1969). This same effect has been found to occur as a placebo reaction in normal human subjects who maintained a relaxed wakeful state during an EEG alpha biofeedback experiment that was considered to be a positive experience.

Rouse and Frank (1974) recently reported that a single small dose of d-LSD-25 (at 1\% of that required to produce consistent overdose symptomology and at most 
$10 \%$ of the direct anticonvulsant dose) given prior to training, significantly facilitated learned resistance to audiogenic seizures in mice. Mainly because of the drug's low-dose effect on learning and memory and because of the well-documented opinion that it does not cause chromosomal damage or other side effects directly attributable to the drug, they suggested the use of this and related compounds as effective and safe adjuncts to current behavioral and electrophysiological biofeedback procedures which are presently being used to treat epilepsy (Poirier, 1974; Sterman \& Friar, 1972) and a variety of other illnesses (Rouse, 1972). The estimated minimally effective dose for such a therapeutic effect was $.1 \mathrm{microgram} / \mathrm{kg}$. This figure is three orders of magnitude below the concentration required to produce nonspecific toxic reactions in blood and brain tissue cultures (Hendelman, 1972; Miura \& Tsujiyama, 1957; Voss \& Winkelhake, 1974). It is also two orders below the maximum concentration capable of enhancing neuronal and glial growth rates (Miura \& Tsujiyama, 1957). It may be noted that even concentrations that do produce increased intracellular lysosomal growth and chromosomal breaks in vitro, and immune response and cell growth rate suppression in vitro do not disturb synaptic or neuropil integrity of cultured brain tissue (Hendelman, 1972). The higher doses that might be required to enhance alpha in the epileptic population in general are therefore far below the toxic level of this compound.

It seems likely that EEG biofeedback training and its applications will benefit from a better understanding of entrainment, drug, and placebo reactions. In this instance, the placebo reaction paralleled the physical effect of the alpha feedback loop because of the following. Mimura et al (1969), reported that intermittent sound stimulation produced a shift from unimodal to bimodal (desynchronization) alpha and then back to unimodality as habituation of the orienting response occurred. It is possible that long-term attending to the intermittent tones produced by the feedback loop entrained alpha through classically conditioned inhibition of the desynchronizing response. If this interpretation is correct, then the feedback loop presenting the tone at the onset of alpha would maximize such learning. Operant control of this response (entrainment or resistance to desynchronization) should then be made more likely because of the increase in baseline entrainment as training progressed. Such training may strengthen attentional mechanisms, the inhibitory effects of which, in animal and human epileptics, are extensively documented although they are still incompletely understood (Guerrero-Figueroa, Barros, \& DeBalbian Verster, 1963). It may also help correct the dissociation of seizure resistance and relaxed wakefulness found in an experimental epileptic preparation (Roldan, Radil-Weiss, \& Chocholova, 1970).

\section{REFERENCES}

Adey, W. R. Spectral analysis of EEG data from animals and man during alerting, orienting and discriminative responses. In C. R. Evans and T. B. Mulholland, (Eds.), Attention in neurophysiology Lond on: Butterworth, 1969, p. 215.

Andersen, P., Junge, K., \& Svet'n, O. Cortico-fugal facilitation of thalamic transmission. Brain, Behavior and Evolution, 1972, 6, $170-184$.

And $170-184$. \& Andersson, S. Physiological basis of the alpha rhythm. New York: A ppleton-Century-Crofts, 1968.

Banquet, J. P. Spectral analysis of the EEG in meditation. EEG Journal, 1973, 35, 143-151.

Beatty, J. Similar effects of feedback signals and instructional information on EEG activity. Physiology and Behavior, 1972, 9, 151-154.

Beatty, J. Effects of initial alpha wave abundance and operant training procedures on occipital alpha and beta wave activity. Psychonomic Science, 1971, 23, 197-199.

Boudrot, R. An alpha detection and feedback control system. Psychophysiology, 1972, 9, 461-466.

Bradley, P. B., Elkes, C., \& Elkes, J. On some effects of LSD-25 in normal volunteers. Journal of Physiology, 1953, 121 50-51.

Brown, B. Subjective and EEG responses to LSD in visualizer and non-visualizer subjects. EEG Journal, 1968, 25, 372-379.

Rown B B Recognition of aspects of consciousness through association with EEG alpha activity represented by a light signal. Psychophysiology, 1970, 6, 442-452.

Brown, B. B. Awareness of EEG-subjective activity relationships detected within a closed feedback system. Psychophysiology, $1971,7,451-464$.

Brumlik, J., Richeson, W. B. \& Arbit, J. The origin of certain electrical cerebral rhythms. Brain Research, 1966/1967, 3 , 227-247.

Chapman, R. M., Armington, J. C., \& Bragdon, H. R. A quantitative survey of kappa and alpha. EEG Journal, 1962, $14,858-868$.

Cooper, R., Shipton, H. W., Shipton, J., Walter, V. J., \& Walter, W. G. Spatial and temporal identification of alpha activities in relation to individual mental states by means of the 22 channel helical scan toposcope. EEG Journal, 1957, 9, 375.

Creutzfeldt, O., Greenewald, G., Simonova, I., \& Schmitz, H. Changes of the basic rhythms of the EEG during performance of mental and visuomotor tasks. In C. R. Evans and T. B. Mulholland, (Eds.), Attention in neurophysiology. London: Butterworth, 1969. P. 148.

Darrow, C. W. Psychological and psychophysiological significance of the electroencephalogram. Psychological Review, 1947, 54, 157-168.

Darrow, C. W.. Vieth, R. N., \& Wilson, J. Electroencephalographic "blocking" and adaptation. Science $1957,126,74-75$.

Delmas-Marsalet, P. Prognostic significance of perfect alpha spindles in essential epilepsy. EEG Journal, 1964, 17, 720. 716-723.

Delucchi, M. R., Garoute, B., \& Aird, R. B. The scalp as an electroencephalographic average. EEG Journal, 1962, 14, 191.

Dewan, E. M. Cybernetics and attention. In C. R. Evans and T. B. Mulholland (Eds.), Attention in Neurophysiology. London: Butterworth, 1969. P. 323.

Engstrom, D. R., London, P., \& Hart, J. Hypnotic susceptibility increased by EEG alpha training. Nature, 1970, 227 , 1261-1262.

Fehmi, L. G. Biofeedback of electroencephalographic parameters and related states of consciousness. Paper presented at the Annual American Psychological Association Convention, Washington, D. C.. 1971 .

Fink, M. Psychopharmacology and EEG. In G. H. Glasser (Ed.), $E E G$ and behavior. New York: Basic Books, 1963. P. 177.

Fink, M. EEG and human psychopharmacology. In H. W. Elliot, W. C. Cutting and R. H. Dreisback (Ed.), Annual review of pharmacology Vol. 19. Palo Alto: Annual Reviews, 1969. Pp. 241-258.

Fink, M. \& Itil, T. M. Neurophysiology of phantastica: EEG and behavioral relations in man. D. H. Efron, (Eds.), Psycho-pharmacology: A Review of Prosress 1957-1967. Washington, D.C: U.S. Government Printing Office, 1968.

Finley, W. W., Smith, H. A., \& Etherton, M. O. Reduction of seizures and normalization of the EEG in a severe epileptic following sensorimotor biofeedback training: Preliminary study. Biological Psychology, in press

Friedlander, W. J. The relation of metrazol EEG-convulsant threshold and alpha index. EEG Journal, 1962, 14, 751-753

Gale, A., Bramby, P., Lucas, B., \& Christie, B. Differential effect of visual and auditory complexity on the EEG negative 
hedonic value as a crucial variable. Psychonomic Science, 1972, 17, 21-24.

Giannitrapini, D. EEG average frequency and intelligence. EEG Journal, 1969, 27, 480-486.

Giannitrapini, D. Stability of EEG components relating to int elligence scores. EEG Journal, 1973, 34, 105.

Giannitrapini, D. Schizophrenia and EEG spectral analysis. EEG Journal, 1974, 36, 377-386.

Gloor. P. Hans Berger on the electroencephalogram of man Electroencephalography and Clinical Neurophysiology, Suppl. No. 28, 1969, Elsevier, Amsterdam.

Goodman, L. S., \& Gilman, G. The pharmacological basis of therapeutics (3rd ed.). New York: Macmillan, 1968.

Green, E. E., Green, A. M., \& Walters, E. D. Voluntary control of internal states: Psychological and physiological. Journal of Transpersonal Psychology, 1970, 2, 1-26.

Guerrero-Figueroa, R., Barros, A., \& De Balbian Verster, F Some inhibitory effects of attentive factors on experimental epilepsy. Epilepsia (Amst.), 1963, 4, 225-240.

Guiti, N. Quantitative analysis of repeated electroencephalograms in the dog under the effects of LSD-25. EEG Journal, 1966, 21, 80-84.

Gullickson, G. R., (Ed.), The psychophysiology of Darrow. New York: Academic Press, 1973.

Hendelman, W. J. A morphological study of the effects of LSD on neurons in cultures of cerebellum. Journal of Neuropathology and Experimental Neurology, 1972, 31. 411-432.

Hernandez-Peon, R., (Ed.), The physiological basis of mental activity. EEG Journal, Suppl. No. 24, Amsterdam: Elsevier. 1963.

Hord, D., \& Barber, J. Alpha control: Effectiveness of two kinds of feedback. Psychonomic Science, 1971, 25, 151-154.

Hord, D., Naitoh, P., \& Johnson, L. Intensity and coherence contours during self-regulated high alpha activity. EEG Journal, 1972, 32, 429-433.

Howe, R. C., \& Sterman, M. B. Somatosensory system evoked potentials during waking behavior and sleep in the cat. EEG Journal, 1973, 34, 605-618.

Itil, T. M. In A. G. Karcznak and W. P. Koella (Eds.), Neurophysiological and Behavioral Aspects of Psychotropic Drugs. Fort Lauderdale, Florida: Thomas, 1969. P. 72.

Jasper, H. H. Neurochemical mediation of specific and nonspecific cortical activation. In C. R. Evans and T. B Mulholland (Eds.), Attention in neurophysiology. London: Butterworth, 1969. P. 377.

Kamiya, J. Operant control of the EEG alpha rhythm and some of its reported effects of consciousness. In C. Tart (Ed.) Altered states of consciousness. New York: Wiley, 1969 P. 489 .

Killam, E. K., \& Killam, K. F. The influence of drugs on central afferent pathways. In W. S. Fields (Ed.), Brain mechanisms and drug action. Springfield: Thomas, 1957. P. 71.

Klir, G. J. Introduction to methodology of switching circuits. New York: Nostrand, 1972.

Kreitman, N., \& Shaw, J. C. Experimental enhancement of alpha activity. EEG Journal, 1965, 18, 147-155.

Legewie, H., Simonova, O., \& Creutzfeld, O. D. EEG changes during performance of various tasks under open- and closed-eyed conditions. EEG Journal, 1969, 27, 470-479.

Lehmann, O. Multichannel topography of human alpha EEG fields. EEG Journal, 1971, 31, 439-449.

Leissner, P., Lindholm, L., Petersen, I. Alpha amplitude dependence on skull thickness as measured by ultrasound technique. EEG Journal, 1970, 29, 392-399.

Lindsley, D. B. Electrophysiology of the visual system and its relation to perceptual phenomena. In M. A. B. Brazier (Ed.) Brain and behavior. (American Institute of Biological Sciences, Washington, D. C.), 1961 . P. 381 .

Lynch, J. J., \& Paskewitz, D. A. On the mechanism of the feedback control of human brainwave activity. The Journal of Nervous and Mental Disease, 1971, 153, 205-217.

Mabry, P. O.. \& Campbell, B. A. Serotonin inhibition of catecholamine-induced behavioral arousal. Brain Research, 1973, 49, 381-391.

Mimura, K., Sato K., Ozaki, T., Honda, M., \& Masuya, S. On the physiological significance of the EEG changes caused by sonic stimulation. EEG Journal, 1962, 14, 683-696.

Miura, T., \& Tsujiyama, $Y$. The effect of psychotropic substances on nerve and neuroglia cells developed in tissue cultures. In S. Garattini and V. Ghetti (Eds.), Psychotropic drugs. Amsterdam: Elsevier, 1957. Pp. 478-482.

Morrell, L. R. Some characteristics of stimulus-provoked alpha activity. EEG Journal, 1966, 21, 552-561.

Mulholland, T. B. Occipital alpha revisited. Psycholggical Bulletin, 1972, 78, 176-182.

Mulholland, T., \& Runnals, S. A stimulus-brain feedback system for evaluation of alertness. EEG Journal, 1962, 14, 233-243.
Nebylitsyn, V. D. Fundamental properties of the human nervous system. New York: Plenum, 1972.

Nowlis, D. P., \& Kamiya, J. The control of electroencephalographic alpha rhythm through auditory feedback and the associated mental activity. Psychophysiology, 1970, 6, 476-484.

Paskewitz, D. A., \& Orne, $\dot{M}$. T. Visual effects on alpha feedback training. Science, 1973, 181, 360-363.

Penfield. W., \& Jasper. J. Epilepsy and the functional anatomy of the human brain. Boston: Little, Brown, 1954. P. 371.

Peper, E. Feedback regulation of the alpha electroencephalogram activity through control of the internal and external parameters. Kybernetik, 1970, 7, 107-112.

Peper, E. Comment on feedback training of parietal-occipital alpha asymmetry in normal human subjects. Kybernetik, $1971,9,156-158$.

Peper E. Localized EEG alpha feedback training: A possible technique for mapping subjective, conscious, and behavioral experiences. Kybernetik, 1972, 11, 166-169.

Peper, E., \& Mulholland. T. B. Methodological and theoretical problems in the voluntary control of electroencephalographic occipital alpha by the subject. Kybernetik, 1970, 7, 10-13.

Perez-Borja, C., Chatrian, G. E., Tyce, F. A., \& Rivers, M. H. Electroencephalographic patterns of the occipital lobe in man: A topographical study based on use of implanted electrodes EEG Journal, 1962, 14, 171-182.

Perry N. W \& Childen, D. G. The human visual evoked response. Springfield, Il: Thomas, 1969. P. 28.

Pfeiffer, C. C., Goldstein, L., Murphree, H. B., \& Sugerman, A. A. Time-series, frequency analysis and electrogenesis of the EEG's of normals and psychotics before and after drugs. The American Journal of Psychiatry, 1965, 121,1147-1155.

Poirier, F. Treatment of epilepsy by learned control of brain waves. EEG Journal, 1973, 34, 736-737.

Pribram, K. J. The neurophysiology of remembering. Scientific American, 1969, 220, 3-13.

Rodin, E., \& Luby, E. Effects of LSD-25 on the EEG and photic evoked responses. Archives of General Psychiatry, 1966, 14, 431-435.

Roldan, E., Radil-Weiss, T. \& Chocholova, L. Paroxysmal activity of hippocampal and thalamic epileptogenic foci and induced or spontaneous changes of vigilance. Experimental Neurology, 1970, 29, 121-130.

Rouse, L. O. d-LSD-25 in the treatment of epilepsy. Paper presented to the Biofeedback Research Society, Boston, 1972

Rouse, L. O. On-line period analysis of EEG by time-to-amplitude conversion (TAC). Psychophysiology, in press.

Rouse, L. O., \& Frank, D. Facilitation of learned resistance to audiogenic seizures in BALB/cCrgl mice by d-LSD-25. Nature, $1974,248,78-81$.

Rubin, M. L., \& Wells, G. L. Fundamentals of visual science, Springfield, Ill: Thomas, 1969.

Sittenfeld, P. The control of the E\&G theta rnytnm. Paper presented to the Biofeedback Research Society, Boston, 1972

Slatter, K. H. Alpha rhy thms and mental imagery. EEG Journal, $1960,12,851-859$.

Stanley-Jones, D. Kybernetics of mind and brain,. Springfield, Ill: Thomas, 1970. P. 140.

Stennett, R. G. The relationship of alpha amplitude to the level of palmar conductance. EEG Journal, 1957, 9, 131-138.

Sterman, M. B. Physiological bases and clinical applications of biofeedback techniques in medicine and psychiatry. VA Symposium, Bedford, MA, $1972 \mathrm{~b}$.

Sterman, M. B. Neurophysiological and clinical studies of sensorimotor EEG biofeedback training: Some effects on epilepsy. In L. Birk (Ed.), Seminars In Psychiatry, 1973, 5 , 507-524

Sterman, M. B., \& Clemente, C. D. Basal forebrain structures and sleep. Acta Neurol-Latinoamer, 1968, 14, 224-228.

Sterman, M. B., \& Friar, L. Suppression of seizures in an epileptic following sensorim otor EEG feedback training. EEG Journal, 1972, 33, 89-95.

Sterman, M. B., Wyrwicka, W., \& Roth, S. R. Electrophysiological correlates and neural substrates of alimentary behavior in the cat. In $J$. P. Morgane and $M$. Wayner (Eds.). Neural regulation of food and water intake. Annals of the New York Academy of Science, 1969, 157, 723-739.

Surwillo, W. W. The relation of response-time variability to age and the influence of brain wave frequency. EEG Journal $1963,15,1029-1032$.

Timmons, B., \& Kamiya, J. The psychology and physiology of meditation and related phenomena: A bibliography. Journal of Transpersonal Psychology, 1970, 1, 41-59.

Timmons, B., Salamy, J., Kamiya, J., \& Girton, D. Abdominal-thoracic respiratory movements and levels of arousal. Psychonomic Science, 1972, 2, 173-175. 
Vogel, W., Broverman, D. M., \& Klaiber, E. L. EEG and mental abilities. EEG Journal, 1968, 24, 166-175.

Vogel, W., Broverman, D. M., Klaiber, E. L., Abraham, G., \& Cone, F. L. Effects of testosterone infusions upon EEGs of normal male adults. EEG Journal, 1971a, 31, 400-403.

Vogel, W., Broverman, D. M., \& Klaiber, E. EEG responses in regularly menstruating and amenorrheic women treated with ovarian hormones. Science, 1971b, 1972, 388-396.

Vogel, W. \& Kun, K. J. EEG response to photic stimulation as a function of cognitive style. EEG Journal, 1969a, 27, 186-190.

Vogel, W., Kun, K. J., Meshour, E., Broverman, D. M., \& Klaiber, E. L. The behavioral significance of EEG abnormality in mental defectives. American Journal of Mental Deficiency. $1969 \mathrm{~b}, 74,62-68$.

Voss, E. W., Jr., \& Winkelhake, J. L. Mechanism of lysergic acid diethylamide interference with antibody biosynthesis.
Proceedings of the National Academy of Sciences, 1974, 71, 1061-1064.

Wallace, R. K., \& Benson, J. The physiology of meditation Scientific American, 1972, 226, 84-90.

Walter, W. G. Can "attention" be defined in psychological terms? In C. R. Evans and T. B. Mulholland (Eds.), Attention in neurophysiology. London: Butterworth, 1969. P. 39 .

Williams, H. L. Granda, A. M., Jones, R. C., Lubin, A., \& Armington. J. C. EEG frequency and finger pulse volume as predictors of reaction time during sleep loss. EEG Journal, $1962,14,64-70$.

(Received for publication February 12, 1974; revision received September $5,1974$. ) 